\title{
BIBLIOGRAFIA PEDAGÓGICA BRASILEIRA (1812 a
}

1900)

No ano de 1935 recomendou o Ministro Gustavo Capanema, à extinta Diretoria Nacional de Educação, fosse levantada a bibliografia pedagógica nacional.

O trabalho foi entregue a uma comissão, composta dos Srs. Joaquim da Costa Ribeiro, Joaquim Moreira de Sousa e Thiers Moreira, e que logo o iniciou, pors-seguindo em buscas na Biblioteca Nacional. Biblioteca Central Pedagógica e Biblioteca Municipal, da Prefeitura do Distrito Federal, c ainda em outras. De cada obra foram extraídas, em fichas, as anotações convenientes. Mas, com a reforma daquela Diretoria c alterações do plano de suas atividades, ficou interrompido o trabalho, até que, em 1941, o Instituto Nacional de Estudos Pedagógicos o retomou, fazendo rever muitas das fichas anteriormente colhidas, e acrescendo-as em novas buscas. Nesta fase do trabalho ocuparam-se a assistente de ensino Selene Tigre Terra e os técnicos de educação Elisa Dias Veloso e Inês Bomilcar Besouchet. Deu, enfim, a apresentação com que agora se publica este estudo retrospectivo a técnica de educação Déa Veioso Barros, com longa experiência cm biblioteconomia, Preferiu-se para esta publicação, considerada ainda como provisória, a apresentação cronológica, a fim de que possa mais facilmente servir aos estudos históricos e, assim também, suscitar as achêgas e observações dos estudiosos no assunto. $O$ Instituto Nacional de Estudos Pedagógicos, que fará publicar, nos próximos. números desta Revista a bibliografia pedagógica brasileira referente ao periodo de 1901 a 1930, c ao de 1931 a 1940, muito agradecerei toda e qualquer contribuição que lhe seja enviada, no sentido de que possa ser completado este estudo, suprido em suas lacunas e corrigido em possiveis enganos. $O$ I.N.E.P. igualmente aceita. Com prazer, a oferta de qualquer livro ou folheto, referente à educação brasileira no passado, para a sua Biblioteca

$$
1812 \text { A } 1820
$$

AMIRADE, Vicente Navarro de - Escola

Médico Cirúrgica. Rio de Janeiro. 1812 Imprensa-Régio. $72 \mathrm{p}$.

Projeto de um instituto de ensino médico, cuja criação o $A$. recomenda para o pais: e de lembrar que o primeiro curso cirúrgico foi criado no ano de 1808 .
1821 A 1830

EDUCAÇÃo - Por Um Brasileiro. Rio de Janeiro. 1822. Imprensa Nacional. 26 p.

Considerações gerais sobre a educação do tempo.

AMERICUS - Cartas Políticas. Londres. 1826. R. Greenlaw. $\left(S / n^{o} p.\right)$.

A XII destas cartas, de au-autoria de Martim Francisco 
Ribeiro de Andrade, apresenta idéias fará um sistema de educação nacional, desenvolvendo a memória que, em 9 de maio de 1823, apresentara à Assembléia Geral Constituinte e Legislativa para a reforma do ensino na província de São Paulo.

LisboA, José da Silva - Constituição Mural e Deveres do Cidadão, conforme o espírito da Constituição do Império. Rio de Janeiro. 1824. Tip. Nacional. 3 vols. c/ 176,163 e $166 p$

Desenvolvido estudo de educação moral e cívica, dedicado à S. M. o Imperador D. Pedro I.

SERPA, Joaquim Jerânimo Tratado de Educação Fisico-Moral ninos. Pernambuco. 1828. dos me "Diário". 196 p.

Crítica ao projeto do deputado l.ino Coutinho, sobre o ensino médico no Brasil.

JobIM, José Martins da Crus - Plano de Organização das Escolas de Medicina do Rio de Janeiro e Bahia. Rio de Janeiro. 1830. Tip. do "Diário". 15 p.

\section{A 1840}

Sociedade Amante da Instrução - Por D. de A. C. Duque Estrada e outros. Rio de Janeiro. 1832. $38 p$.

Relatório das atividades da instituição referida no ano de 1831.

Cartas sobre a educação das meninas - Trad. de João Cândido de Deus e Silva. Rio de Janeiro. 1838. Tip. Nacional. 245 p.
Adaptação de obra de autora americana, cujo nome se declina.

Degerando, Barão de - Curso Normal para Professores de Primeiras Letras. Trad. de Dr. João Cândido de Deus e Silva. Niterói. 1839. Tip. Niterói de M. G. de S. Rego.

Orientação para o ensino normal; a tradução é acompanhada das leis gerais e provinciais sobre o ensino primário da época.

\section{A 1850}

RocHA, Justiniano José da - Instrução Pública. Rio de Janeiro. 1842. Tip. Universal Laemmert. 27 p.

Relatório sobre a situação do ensino na província do Rio de Janeiro.

BRASILEIRA, Augusta Nisia floresta Conselhos a minha filha. $2^{\circ} \mathrm{Ed}$. Rio de Janeiro. 1845. F. de Paula Brito. 3 p.

Exortação à juventude, para a prática da modéstia e da virtude.

UbAtubA, Manoel Pereira da Silva Algumas considerações sobre a educação física. Niterói. 1845. Tip. Comercial. $29 p$.

Tese apresentada à Faculdade de Medicina do Rio de Janeiro.

Melo, Joaquim Pedro de - Generalidades acerca da Educação Física dos Meninos. Rio de Janeiro. 1846. Tip. Teixeira \& Cia. $\left(S / n^{o}\right.$ p.)

Tese apresentada à Faculdade de Medicina do Rio de Janeiro. 
instrução Pública - Pelo Conselho de Instrução Pública. Bahia. 1846. Tip. de Galdino Bezerra \& Cia $\left(S / n .^{o}\right.$ p.) Relatório apresentado à Asscmhlcia Legislativa da Província da Bahia.

Mota, Peruando Sebastião Dias da Instrução Pública. Rio de Janeiro. 1846. Tip. do "Diário" de N. L.

Viana. $16 \mathrm{p}$

Relatório sobre o estado do ensino na Província do Rio de Janeiro.

CAJueIro, Inácio Hermógenes - $\mathrm{O}$

Educador da Mocidade Brasileira. Bahia. 1P48. S/Edit. 4 p.

Explicação didática de trechos extraídos da Bíblia.

FREEZE. João Henrique - Noções gerais acerca da educação da mocidade brasileira. Rio de Janeiro. 1850. E \& Laemmert $68 p$

Principios sobre educação. encarada sob o ponto de vista físico, moral, intelectual, reli-gioso.

REBELo, Guilherme Pereira (Dr.) - Instrução Pública. Sergipe. 1851. Tip, Província de Sergipe. $22 \mathrm{p}$.

Relatório da inspeção geral das aulas públicas da Província de Sergipe.

MoraIS. Alexandre J. Melo (Dr.) - $\mathrm{O}$ Educador da Mocidade Brasileira. Bahia. 1852. Epifanio Pedrosa. $84 p$. Texto de lições morais.

AMERICANA. Zaira - Educação da mulher. Rio de Janeiro. 1853. Tipografia Dois de Dezembro, 390 p.
Estudo sobre as vantagens que decorrem, para a sociedade, da educação da mulher.

1851 A 1860

Portugal, Antônio Nunes de Gouveia

- Influência da Educação Física

do Homem. Rio de Janeiro. 1853.

Tip. Universal de l.aemmert. $27 p$ ene.

Tese apresentada a Fatulda-de de Medicina do Rio de Janeiro.

XAVIER. Inácio Firmo - Reflexões sobre a Educação Física e Moral da Infância. Recife. 1854. Ti. Universa/. (S/n. ${ }^{o}$ p.)

Escritos de propaganda.

AZEVEDO, Joaquim Gonçalves (cônego)

- Instrução Pública. Amazonas.

1855. M. S. Ramos. $7 p$.

Relatório sobre a situação da instrução pública na Província do Amazonas.

Borges, Abílio César — Instrução Pública. Bahia. 1856. S/Edit. 71 p. Relatório sobre a situação do ensino na Província da Bahia.

SilveIRA, Manoel Lourenço da - Instrução Publica. Pernambuco. 1857. Tip. de Manuel Figueiroa de Faria. 32 p.

Relatório sobre a situação da instrução pública na Província de Alagoas.

BRAsiL, Tomás Pompeu de Sousa (Dr.)

- Relatório do estado da Instru ção Pública na Província do Cea rá no ano de 1857. Ceará 1858. Tip. Cearense. 75 p.

Faculdade de Medicina do Rio de Janeiro. Memórias Históricas. Rio 
REVISTA BRASILEIRA DE ESTUDOS PEDAGÓGICOS

de Janeiro. 1857-1879. S/Edit. 18 vols.

Memórias históricas dos anos de 18S5 n 1878 apresentados à Congregação de Lentes da Faculdade FigueIREdo, Carlos Honorio de - Faculdade de Medicina do Rio de Janeiro.

Programa do Ensino da Instrução Secundária do Município da Corte

- Rio de Janeiro. 1858. Tip. $\mathrm{Na}$ cional $\left(S / n .^{\circ} p.\right)$

SARMEnto. Casimiro José de Morais - A Educação Física de Meninas. Rio de Janeiro. 1858. Tip. Universal de Laemmert. $176 \mathrm{p}$.

Ensaios de princípios gerais da educação, entre ns quais se salienta o respeito devido à personalidade criança.

PACHECO, J. Praxedes P. (Br.) - Brasilismo. Rio de Janeiro. 1858. J. Villeneuve \& Cia. 48 p.

Notas de um estudioso, sobre como se deve educar a família .

Faculdade de Medicina da Bahia - Memórias Históricas. Bahia $c$ Rio de Janeiro. 1858-1880. S/Edit 10 vols.

Memórias históricas dos acontecimentos mais notáveis dos anos de $1857,58,61,63,66,68,77,78,79$,

Oliveira, João José Barbosa d' (Dr.)

- Instrução Pública. Bahia. 1858.

S/Edit. (S/n. ${ }^{o}$ p.)

Relatório sobre o movimento do ensino na Província da Bahia.

MotA, Joaquim Inácio Silveira, da (Dr.)

- Instrução Pública. Curitiba.

1858. Cândido Martins Lopes. 64 p.
Relatório sobre a situação da Instrução Pública da Pro-vincia do Paraná. Instituto Histórico". vol. XXII. p. 507 a 526.

Memória sobre a fundação das $\mathrm{Fa}$ culdades de Direito do Brasil.

PInTo, Diogo de Mendonça - Instrução Pública. São Paulo. 1859. Antônio Lousada Antunes. ( $S / n .{ }^{\circ}$ p.).

Relatório sobre o movimento da instrução pública na Pro-vincia de São Paulo, no ano anterior.

REBOLÇAS, $M . M$. - Tratado sobre a Educação Doméstica e Pública. Bahia. 1859. Antônio O. da F. Guerra. 343 p.

Principios e regras de puera cultura.

\section{A 1870}

OliveirA, João José Barbosa d' (Dr.) Instrução Pública. Bahia. 1861. S/Edit. (S/n. $\left.{ }^{o} p.\right)$.

Relatório sobre o movimento do ensino na Bahia no ano de 1860.

Pacheco, J. Praxedes - O ensino Praxedes. Rio de Janeiro, 1861.

$$
\begin{aligned}
& \text { Compêndio de didática antiga } \\
& \text { para ensino das matérias do } \\
& \text { currículo primário da época. }
\end{aligned}
$$

Regulamento geral e programas do ensino e horários das escolas normais primárias. Bahia. 1861. Tip. A. D. da França Guerra. 24 p. c/ I mapa.

RAULICA, Ventura de (Padre) - Estudo sobre o ensino público. Trad. 
de Aprígio Justiniano da Silva Guimarães. 2. ${ }^{\text {a }}$ Ed. Recife. 1861. Geraldo Henrique de Mira \& Cia.

\section{ns p.}

Discurso sobre a necessidade de uma reforma do ensino público, no interesse da religião; o A. condena o esludo dos clássicos.

Bastos, A. C. Tavares (Dr.) - Cartas do solitário. 2. ${ }^{a}$ Ed. Rio de Janeiro. 1836 Tip. da Atualidade.

Dois capitulos (24 pgs.) sobre o ensino religioso.

Pacheco, J. Praxedes Pereira - Verdadeira Educação Cívica Popular, proporcionada em conferências. Rio de Janeiro. 1863. S/Edit. $\left(S / n .^{o} p.\right)$

Lições ao povo, sobre moral, religião e civismo.

FRAZÃo, Manuel José Pereira - Cartas do Professor da Roça. Rio de Janeiro. 1864. Tip. Paula Brito. 44 p.

Artigos relativos ao ensino na Corte, publicados no " Constitucional", de março e abril de 1863.

Assis, Joaquim José de — Instrução Pública. Pará. 1864. Fred. C. Rhorsard. (S/n. ${ }^{\circ}$ p.)

Relatório sobre a situação do ensino na província do Grão Pará.

Daugault - Curso Prático de Pedagogia. Trad. de Joaquim Pires Machado Portela. Recife 1865. Tip. Universal. 186 p.

Primeira obra de exposição metódica do assunto publicada no país.
Coleta para Educação de Missionários - Rio de Janeiro. 1865. A Gon çalves Guimarães \& Cia. 15 p.

Obra publicada sob o patrocinio de SS. MM. o Imperador e a Imperatriz do Brasil, acerca do aproveitamento de vocações para o estado eclesiástico e criação de bolsas de estudo.

BARroso, José Liberato (Conselheiro)

- A Instrução Pública no Brasil. Ria de Janeiro. 1867. B. L. Garnier. $265 p$.

Dados históricos acompanhados de comentários sobre a situação do ensino no Brasil, nos seus diferentes graus.

Abreu, Eduardo Augusto Pereira de (Dr.) Educação Física, Intelectual e Moral do Soldado. Rio de Janeiro 1867. Esteves \& lrmão. $44 p$.

Principios relativos à escolha $e$ formação de pessoal para a boa organização do Exército Nacional.

ESTRADA, D. de A. C. Duque — Imperial Sociedade Amante da Instrução. Rio de Janeiro. 1867. Tip. Perseverança. 36 p.

Histórico da criação e relatório das atividades da associação referida.

NÜRINEIXY, Luiz José de - Imperial Sociedade Amante da Instrução. Rio de Janeiro. 1868. Tip. Perseverança. 40 p.

Considerações acerca da importância dos trabalhos da sociedade que dá título ao trabalho . 
Monteiro. Joaquim dos Remédios (Dr.) Higiene e Educação da Infância. Resende (Est. ao Rio) 1868 Fernandes \& Cia. 82 p.

Sugestões e conselhos práticos sobre a educação fisicica das crianças.

MOREIRA, Nicolau Joaquim - A Educação Moral da Mulher. Rio de Janeiro. 1868. Tip. Nacional. 14 p.

$$
\begin{gathered}
\text { Discurso pronunciado } \\
\text { Academia Imperial de Mediana }
\end{gathered}
$$

DUPIN - Reflexões sobre o ensino e o estudo do direito. Trad. e anot. por J. J. de Campos da Costa Medeiros $c$ Albuquerque. 2. ${ }^{\text {a }}$ Ed. Recife. 1868. Tip. Universal $79 p$.

Exposição de princípios e regras a serem aplicados ao en-sino jurídico.

ALMEIDA JÚNIOR. Manuel Ribeiro Liberdade do Ensino. Rio de Janeiro. 1868. Tip. do Correio Mercantil. $17 \mathrm{p}$.

Considerações gerais sobre as vantagens do ensino livre.

Bastos, Tavares - A Província. Parte III Interesses Provinciais. Cap. I- pg. 227 a 254. Rio de Janeiro. 1870. ti. L. Garnier. 418p

$O$ A. apresenta nesse capitulo idéias sobre liberdade e obrigatoriedade do ensino, taxa escolar $c$ organização do ensino nas provincias.

Ministério da Instrução Pública na Prússia Novo Guia para o ensino da ginástica nas escolas públicas da Prússia. Trad. ordenada pelo Ministro do Império. Rio de Janeiro. 1870. Tip. do Imperial
Instituto Artístico. 158 p. c/ 33 fig.

Exposição de jogos educa tivos para serem usados nas escolas primárias.

SouZA, Paulino José Soares de - Instrução

Pública. Rio de Janeiro.

1870. Tip. Nacional. $24 p$.

Projeto apresentado à Cà-mara dos Deputados na sessão de 6-81870, propondo a criação de uma universidade na capital do Império, bem como de um Conselho Superior de Instrução Pública.

\section{A 1880}

MACEDO, Joaquim Teixeira de - A Instrução Pública na Prússia. Rio de janeiro. 1871. Tip. Nacional. 311 p.

Estudos sobre a legislação e organização da instrução na Prússia.

Instrução Pública - Por diversos professores públicos. Rio de Janeiro.

1871. Júlio Villeneuve \& Cia. $21 \mathrm{p}$.

Manifesto dos professores públicos de Instrução Primária da Corte ao Imperador, ao primeiro Ministro do Império, sobre a situação de penúria em que viviam.

Oliveira, Fernandes de - Ensino Obrigatório. Rio de Janeiro. 1871. Tip. Perseverança. $36 p$.

Discursos pronunciados na Assembléia Provincial Legislativa de São Paulo, sobre a necessidade da obrigatoriedade escolar no país. 
Borges, Abílio César - O Colégio Abílio. Rio de Janeiro. 1872. Imperial Instituto Artístico. $\left\{S / n .^{o} p\right.$. $\}$

Plano de estudos e estatutos do Colégio Abílio. fundado na Corte do Império.

Monteiro, Joaquim dos Remédios (Dr.) O

Ensino Obrigatório. Santa Catarina. 1872. Tif. de J. J. Lopes. $18 p$.

Carta aos membros da Assembléia Provincial de Sta. Catarina, na qual se advoga o ensino obrigatório.

SouzA, A. Nunes de e Oliveira, A. de Almeida - Conversas Públicas na Escola Normal. Maranhão. 1872. Tip. do-País". 39 p.

Duas conferências, de que a primeira tem o titulo " Instrução e Ignorância"

Belfort, J. J. Tavares - A criação de uma Universidade no Brasil. Pernambuco. 1873. Tip. Mercantil. $191 \mathrm{p}$.

Parecer sobre o projeto de criação de uma Universidade no Brasil, da autoria do Conselheiro João Alfredo Correia de Oliveira.

CRuZ, Vicente Ferreira Machado da Educação Doméstica. Resende (Est. do Rio). 1873. S/Edit. 36 p.

Carta dirigida às mães sobre a necessidade da educação doméstica.

LeItão, Antônio Cândido Cunha - Ensino Livre. Rio de Janeiro. 1873. L. Winter. $26 p$.

Projeto de lei apresentado $d$ Câmara dos Deputados sobre o ensino partícula).
Comissões reunidas de Instrução Pública - Imperial Instituto dos Meninos Cegos. Rio de Janeiro.

1873. Tip. Nacional (S/n. ${ }^{\circ}$ p.)

Projeto de lei apresentado à Càmara das Deputados na ses-são de 18-8-1873, sobre a or-ganizacão do referido Instituto.

ARMonde, . A. F. M. — Da Educação Física, intelectual e moral da mocidade no Rio de Janeiro. Rio. 1874, Tip. do Apóstolo. 32 p.

Branco, F. Gil Castelo - Instrução Pública. S/Local. 1874. S/Edit. 144. p.

Relatório sobre a situação do ensino na Província do Rio de Janeiro.

Daligault - Curso Prático de Pedagogia. Trad. de Joaquim Pires Machado Portela. Rio de Janeiro.

1874. A. A. da Crus. (S/n. ${ }^{\circ}$ p.).

Nova tradução desta obra, publicada primeiramente em 1865.

LeITe, Nicolau França - Educação Pública. São Paulo. 1874. Tip. Americana. (S/n. ${ }^{\circ}$ p.).

Conferências de propaganda.

Matos, João Wilkens de - Instrução Pública. Rio de Janeiro. 1874, Tip. Nacional $\left(S / n{ }^{o} p\right.$.).

Relatório sobre o estado da instrução pública do Pará,

Moura, Francisco - As Conferências Populares no Brasil. (Iniciativa do Sr. Cunha Leitão). Rio de Janeiro. 1874 Pinheiro \& Cia. $16 \mathrm{p}$.

Dados históricos e comentários sobre a situação do ensino no país. 
REVISTA BRASILEIRA DE ESTUDOS PEDAGÓGICOS

Oliveira, A. de Almeida - O Ensino Público. Maranhão. 1874. M. V. V. Pires. $472 \mathrm{p}$.

Situação em que se achava o ensino público no Brasil e sugestões para sua completa re-forma.

PessoA., Cirilo - Reorganização do Ensino na Bahia. Rio de Janeiro 1874. Tip. Comercial. $128 p$

Informações relativas à reforma do ensino na Província da Bahia.

Plano Nacional de Educação de 1826 - Por Januário da Cunha Barbosa. José Cardoso Pereira de Melo e Antônio Ferreira França. Rio de Janeiro. 1874. Tip. Imperial Instituto Artístico. (S/n. p.)

Reprodução do projeto apresentado pela Comissão de Instrução Pública da Câmara dos Deputados, em 16 de junho de 1826. Esse projeto, que não leve andamento, dividia a ins-trução pública em quatro graus: pedagogia, liceus, ginásios c academias. A denominação "plano nacional de educação" c desta publicação e não do trabalho original, que vem reproduzido na obra de Primitivo Moacir, "A Instrução c o Império". 1. ${ }^{\circ}$ vol., p. 149 e seg., (Brasiliana, vol. 66. Ed. Nacional, S. Paulo, 1936)

Silva JúNIOR, Pacheco da-O colégio Pedro II. Rio de Janeiro. 1874. Impr Industrial de J. P. Ferreira Dias. 29 p.

Estudo sobre o referido es tabelecimento, seu passado, pre sente efuturo.
Reorganização do Ensino Público em Pernambuco - Recife. 1874. M. Figueiroa \& Filhos. 160 p.

Leis c regulamentos do ensino público na Província de Pernambuco.

CAvalcante, João Barbalho Uchoa Instrução Pública. Recife. 1875. Tip. de Manuel Figueiroa de Faria \& Filhos. ( $S / n{ }^{o}{ }^{\circ}$ p.).

Relatório sobre a situação da instrução pública na Província de Pernambuco.

CRUZ, Guilherme Francisco - Colonização e Ensino Popular. Pará. 1875. Tip. da Constituição. $76 \mathrm{p}$.

Subsidios para a história do ensino público no Pará; crítica à equiparação das escolas normais aos liceus ou ginásios.

Macedo, Joaquim Teixeira de - $O$ Ensino Primário na Prússia. Rio de Janeiro. 1875. Tip. Nacional. $189 p$.

Exposição pormenorizada da organização do ensino público na Prússia.

REIS, Aarão Leal de Carvalho - $\mathrm{A}>$

Instrução Superior do Império. Rio de Janeiro. 1875. Domingos I.uis dos Santos. $91 p$

Coletânea de artigos publicados na imprensa da Corte.

BARroso, José Liberato - A Educação da Mulher. Rio de Janeiro. $1876 . \quad \mathrm{J}$. Villeneuve \& Cia 24 p.

Conferências populares.

Borges, Abilio César - Vinte anos de propaganda contra a palmatória e outros meios aviltantes no ensino da mocidade. Rio de Janeiro 1876. Tip. Cinco de Março. 46 p. 
Coletânea de escritos do autor, contra o uso do castigo físico nas escolas.

CARMo, José Joaquim do - A reforma da Instrução Pública. Rio de Janeiro. 1876. Tip. 5 de Março. 187 p.

CORREIA, Manuel Francisco (Cons.) Concorrência do elemento municipal a bem do ensino. Rio de Janeiro. 1876. J. Villeneuve \& Cia. 12 p.

Exposição de como as municipalidades poderiam concorrer em favor do ensino.

CORREIA, Manuel Francisco (Cons.) - A Educação na Família e na Escola. Rio de Janeiro. 1876. J. Villeneuve \& Cia. $44 p$

\section{Conferência.}

CORReIA, Manuel Francisco (Cons.) - Ensino Obrigatório. Rio de Janeiro. 1876. /. M. de Oliveira c $H$. Chaves. 13 p.

Conferência em que o A. discute se as assembléias provinciais, na legislação do enst no, podem impor penas administrativas $c$ disciplinares por (atos ou omissões não previstos Código Penal.

CorreiA, Manuel Francisco (Cons.) - O Ensino Moral. Rio de Janeiro. 1876. S/Edit. $12 p$.

\section{Conferência.}

FERrEIRA, Fclix - Do Ensino Profissional. Rio de Janeiro. 1876. Imprensa Industrial. $237 p$.

Subsídios para a história do Liceu de Artes e Ofícios do Rio de Janeiro, com notas sobre o ensino técnico profissio- nal $\mathrm{c}$ de belas artes cm vários países da Europa.

LeITE, Fobias R. (Dr.) — Instituto dos SurdosMudos do Rio de Janeiro. Rio de Janeiro. 1876. Tip. Universal de E. de $H$. Laemmert. 16 p.

Noticia do Instituto dos Surdos Mudos do Rio de-Janeiro enviada para a Exposição de Filadélfia com os artefatos de seus alunos.

MACEDO, Joaquim Teixeira de - Ensino Normal Primário e Educação Popular. Rio de Janeiro. 1876. João A. A. d'Aguiar. $\left(S / n .{ }^{\circ} p.\right)$

Breves apontamentos para estudo das questões relativas ao ensino normal, primário e educação popular, coligidos de várias publicações.

MACEDo, Joaquim Teixeira de (Dr.) Ensino Primário. Rio de Janeiro. 1876. M. A. A. d'Aguiar. 233 p.

Apontamentos para estudo das questões relativas ao ensino normal e à educação popular, coligidos de várias publicações cm língua alemã.

RIO BRANCO, (Visconde de) - Escola Politécnica. Rio de Janeiro. 1876. Tip. Universal. $\left(S / n{ }^{\circ}\right.$ p.)

Relatório apresentado pelo diretor ao Ministro do Império .

CARvalho, José Eduardo Freire — Instrução Pública. Bahia. 1877. Tip. do Correio da Bahia. 18 p. e 14 mapas.

Relatório sobre a situação do ensino na Bahia. 
DÓRIA. Franklin - A Instrução. Rio de Janeiro. 1877. Tip. Nacional. $15 p$.

Discursos pronunciados na Câmara dos Deputados sobre a reorganização do ensino primário $e$ o ensino livre superior.

LEITÃo. Antônio Cândida da Cunha Liberdade do Ensino Superior. Rio de Janeiro 1877. J. Villeneuve \& Cia. 14 p.

Discurso proferido na Câmara dos Deputados em prol da liberdade ampla do ensina superior.

MACEDo, Joaquim Teixeira de (Dr.) -- Breves Apontamentos para o Estudo das Questões relativas ao Ensino Normal, Primário c à Educação Popular. Rio de Janeiro, 1877. João M. A. A. d'Aguiar. 233. $p$.

Excertos de várias publicações de autores alemães a rei peita do ensino primário.

Brasil, Carlos Augusto Soares - Compêndio de Pedagogia. Rio de Janeiro. 1878. Tip. Fluminense. $\left(S / n .^{o}\right.$ p.).

Parece ser a primeira obra de exposição sistemática do assunto, devida a autor nacional; trata dos princípios, métodos e objeto da educação.

Costa e Cunha, Estevão da - Memória sobre as Escolas Normais. Rio de Janeiro. 1878. Tip. Nacional. 28 p.

\section{Estudo histórico.}

MotA, Joaquim Inácio Silveira da - Instrução Pública c Educação Na cional. Rio de Janeiro, 1878. Dias da Silva Júnior. 174 p.

Conferências.

Rio BrAnCO, (Visconde de) - Escola Politécnica. Rio de Janeiro. 1878. Tip. Nacional. ( $S / n{ }^{\circ} p$.).

Relatório da diretoria da es- cola, apresentado ao Governo Imperial em março de 1878.

Carvalho, Carlos Leôncio de - O Ensino Primário e Secundário no Município da Corte e o Superior em todo o Império. Rio de Janeiro. 1879. Imp. Nacional. 22 $p$.

Reforma aprovada pelo Dec. $n^{o}$ 7.247, de 19/4/1879.

Figueiredo, José Bento da Cunha (Cons.) Instrução Pública. Rio de Janeiro. 1879. Tip. Nacional. 68 p.

Relatório sobre a situação do ensino primário $c$ secundário no município da Corte.

Instrução Pública - Recife, 1879. M. Figueiroa \& Filhos. 174 p.

Conferências pedagógicas feitas em abril de 1878, no Recife. Publicação ordenada pelo Governo Provincial.

MACEDO, Joaquim Teixeira de (Dr.) - A Escola de Froebel e os Jardins de Infância. Rio de Janeiro. 1879. Tip. Nacional. 2 p.

Ensaio sobre a educação préescolar.

CAvalcanti, João Barbai lio Uchoa Instrução Pública. Pernambuco 1880. Tip. de M. Figuciroa \& Cia. 64 p.

Relatório sobre o ensino primário, normal c secundário da Província. 
REVISTA BRASILEIRA DE

LeITE, Tobias (Dr.) - Instituto dos Surdos Mudos do Rio de Janeiro. Rio de Janeiro. 1880. Tip. Universal de E. H. Laemmerl. $89 \mathrm{p}$.

Relatórios sobre a vida do Instituto apresentados pelo seu Diretor, nos anos de 1877, 78, $79 \mathrm{c}$ 80 .

LIMA, Afonso Hcreulano - Educação Nacional. Rio de Janeiro. 1880. Tip. da "Gazeta de Noticias". 50 p.

Coletânea de artigos $\mathrm{cm}$ que o $A$. se insurge contra os estudos apressados, empenhando-se pela cultura sólida dos discípulos.

MACEDO, Joaquim Teixeira de - Questões relativas à Educação Nacional. Rio de Janeiro. 1880. Tip. Nacional. 291 p.

Exposição e crítica da legislação alemã sobre as escolas normais $e$ jardins da infância.

SANTA ISABEL (Visconde de) — Faculdade de Medicina. Rio de Janeiro. 1880. Tip. Nacional. $15 \mathrm{p}$.

Crítica à legislação da Faculdade de Medicina do Rio de Janeiro.

\section{A 1890}

FEI.IX, Ferreira - A Educação da Mulher. Rio de Janeiro. 1881. Tip. Hil-debrant. 52 p.

Coletânea de notas e pensamentos.

LEITE, Tobias - Ensino dos Surdos--Mudos. 3. ${ }^{a}$ Ed. Rio de Janeiro. 1881. Tip. I.aemmert \& Cia. 400 p.

Compendio para o ensino dos surdos-mudos.

Borges, Abilio César (Barão de Macaú-bas) — Dissertação Pedagógica. Rio de Janeiro. 1882. Tip. do "O Cruzeiro".

$15 p$

Trabalho lido no $1^{\circ}$ Congresso Pedagógico Internacional, em Buenos Aires, a 2 de maio de 1882.

CAVAlCANTI, João Barbalho Uchoa Instrução Pública. Recife. 1882. Manuel Figueiroa de Faria e Filhos. (S/n. ${ }^{o}$ p.).

Relatório sobre o ensino na

Província de Pernambuco.

Congresso Pedagógico Internacional da América do Sul. Rio de Janeiro. 1882. Tip. do "O Cruzeiro". 30 p.

Juízo da Imprensa do Rio da Prata sobre o Delegado do Império do Brasil, o Sr. Abilio César Borges, Barão de Macaú-bas, e o trabalho lido pelo mesmo, nesse certame.

Mendes, Teixeira - A Universidade. Rio de Janeiro. 1882. Tip. da "Gazeta de Notícias". $70 \mathrm{p}$.

Coletânea de artigos sobre a organização universitária.

Reforma do Ensino Secundário e Superior Parecer c projeto (relativo ao Decreto $n .^{\circ}$ 7.247, de 19/4/1879) apresentado pela Comissão de Instrução Pública da Câmara dos Deputados, composta dos Srs. Rui Barbosa (Relator), Tomas de Bonfim Spínola e Ulisses Machado Pereira Viana. Rio de Janeiro. 1882. Tip. Nacional. 114 p.

Análise e crítica do projeto de lei referido, e que conclui pela apresentação de um substitutivo. Depois de considerações gerais sobre a liberdade do ensino superior, que a Comissão advoga, c examinado o problema do en- 
sino secundário, considerado à luz dos mais modernos principios. $O$ projeto é contrário à bifurcação dos estudos secundários; propõe para ele a obrigatoriedade do ensino cientifico; estabelece que além do curso de bacharelado proporcione o ensino secundário seis outros cursos. de finanças, comércio, agri-mensura. máquinas, industrial, relojoaria $e$ instrumentos de precisão. Quanto ao ensino superior, o parecer emenda o projeto nos cursos de medicina a que acrescenta cadeiras; aplaude a inicializa deste, quanto a permitir-se o ensino médico para o sexo feminino; propõe que se substitua, no curso de direito, o ensino de "direito natural", pelo de sociologia; para o curso da escola politécnica, então preparatória de escola de engenharia civil, aponta várias modificações, inclusive o estudo da química orgânica c o estudo da fotografia; propõe a criação de um curso de engenheiros eletricistas. Para a escola de engenharia civil, como para a escola de minas, indica várias modificações do plano de estudos. $O$ projeto examina também o curso superior de ciências físicas e naturais do Museu Nacional e propõe a criação do ensino agronômico, declarando que, "se o Brasil é um país essencialmente agrícola, por isso mesmo cumpre que seja um país ativamente industrial". Pelas considerações, que emite, observações sabre a vida do tempo e abundante informação de educação comparada, é este um dos mais notáveis documentos para o estudo da evolução cultural do país. Sem valor histórico é acrescido pelo apêndice $\mathrm{cm}$ que se transcrevam todos os projetos sobre instrução pública, apresentados à Câmara dos Deputados de 1870 a 1880. (Reeditado em 1942, pelo Ministério da Educação, na cot. " Obras Completas de Rui Barbosa", vol. IX. Tomo 1).

BandeIRA Filho, A. H. de Sousa (Dr.) - O Jardim Infantil na natureza, seu fim e seus meios de ação. Rio de Janeiro. 1883. Tip. Nacional. $92 \mathrm{p}$.

Relatório sobre a organização dos jardins de infância na frança, Áustria, Alemanha e Suiça.

Borges, Abílio César - A Lei do Ensino Infantil. Rio de Janeiro. 1SS3. Tip. I 'uizrrsal. $30 \mathrm{p}$.

Estudo sabre os principios cientificos e humanos de uma nova pedagogia, com relação à individualidade da criança.

Celso, Afonso (Cons.) - Algumas idéias sobre a instrução primária e secundária. Rio de Janeiro. 1883. Tip. Nacional. 92 p.

$O$ autor discorre, larga e documentalmente. sobre os seguintes assuntos: criação de um fundo escolar na Corte c nas Províncias; competência dos Poderes (ierais para criar estabelecimentos de ensino; condições do ensino obrigatório no Brasil.

DiÉGUER JÚNIOR, Manuel Baltazar Pereira Liberdade de Ensino. Maceió. 1883. Amintas de Mendonça. 8 p.

Conferência em prol da liberdade ampla do ensino. 
Ensino Superior e Secundário - Por uma Comissão da Escola Politécnica. Rio de Janeiro. 1SS3. Tip. Nacional. 14 p.

' Parecer sobre o projeto de reforma do ensino superior $e$ secundário.

Reforma do Ensino Primário e várias instruções complementares da Instrução Pública - Parecer c Projeto da Comissão de Instrução Pública da Câmara dos Deputados composta dos Deputados Rui Barbosa, Tomaz de Bonfim Spinola $e$ Ulisses Machado Pereira Viana. Relator: Rui Barbosa. Rio de Janeiro. 18S3. Tip. Nacional. 378 p. 7 graf.

Análise e critica do projeto relativo ao Decreto $n .^{\circ}$ 7.247. de 19/4/1879, na parte relativa ao ensino primário, e que conclui pela apresentação de um substitutivo sobre a matéria.. $O$ parecer compreende 18 longos capítulos, versando o ensino primário e o ensino normal sob os seus mais variados aspectos. desde os filosóficos e politicos até os de reforma dos processos de ensino, parte em que desce. por vezes, à minudenciação exaustiva. Talvez seja este um documento parlamentar único no mundo, pela profundeza do estudo, riqueza de informações sabre a educação do tempo, nos países mais adiantados na época, c o ensino no país. Muitas das idéias defendidas pelo parecer c projeto substitutivo, que não teve, aliás, andamento na Câmara. foram consagradas, depois, pela legislação ou pelo uso, nas escolas brasileiras, consti-Iruindo outras pontos pelos quais ainda se batem os renovadores do ensino $\mathrm{cm}$ nossos dias. O parecer salienta a importância da estatística escolar, a necessidade da criação de um Ministério da Instrução Pública, e $a$ de se estabelecerem planos de financiamento da'educação; estuda profundamente a questão da obrigatoriedade escolar, a do ensino leigo e a da liberdade do ensino; analisa os métodos e programas escolares, fornecendo valiosos subsidios sobre a didática de cada disciplina; examina ainda, além de outras, as questões da organização e administração pedagógica, a formação do professorado, $c$ os problemas de higiene escolar. Podo o trabalho é fundamentado eni rica bibliografia sobre a educação inglesa, iwrte-amcrliana. francesa $i$ alemã da época.

Silva, Domingos Carlos (Dr.) — Da Reforma do Ensino Superior no Brasil. Bahia. 18S3. Impr. Econômica. 120 p.

Estudos sobre o ensino superior no Brasil e suas necessidades, sugestões para uma reforma.

Atas e Pareceres do Congresso da Instrução Rio de Janeiro. 1884. Tip. Nacional.

Embora não se tenha realizado o Congresso de Instrução, mas simplesmente uma Exposição Pedagógica, publicaram-se as memórias e pareceres, sabre os vários temas do programa, os quais se apresentam neste volume. de 976 ps., com numeração própria de cada trabalho. Vão estes relacionados a seguir: 
Amarante, Tarquinio B. de Sousa Competência dos podarei provinciais para criar estabelecimentos de ensino superior. In "Atas e Parccercs do Congresso de Instrução do Rio de Janeiro" — S p. Rio de Janeiro. 1884. Tip. Nacional.

Amorim, João Pires de (Cônego) - Ensino e Religião nas Escolas Primá-rias, Secundárias e Normais. In "Atas e Pareceres do Congresso de Instrução do Rio de Janeiro" - 8 p. Rio de Janeiro. 1884. Tip. Nacional.

Andrade, Boaventura Plácido Lameira - Organização e atribuições do con selho diretor da instrução primária e secundária do município da corte. In "Atas e Pareceres do Congresso de Instrução do Rio de Janeiro" - 2 p. Rio de Janeiro. 1884. Tip. Nacional.

Andrade, Maria Guilhermina Loureiro de Organização dos Jardins da Infância. In "Atas e Pareceres do Congresso de Instrução do Rio de Janeiro "

- 2 p. Rio de Janeiro. 1884. Tip. Na cional.

Andrade. Nuno de - Liberdade do ensino superior. In "Atas e Pareceres do Congresso de Instrução do Rio de Janeiro" - 8 p. Rio de Janeiro. 1884. Tip. Nacional.

Aquino, João Pedro de (Dr.) - Educação Física nos Jardins de Infância, nas Escolas Primárias e Colégios. In " Atas e Pareceres do Congresso de Instrução do Rio de Janeiro " -7 p. Rio de Janeiro. 1884. Tip. Nacional.

Araújo, Antônio Bahia da Silva - Liberdade de Ensino Primário e Secundário. Ensino Obrigatório. Meios de torná-lo efetivo In "Atas e Pareceres do Congresso de Instrução do Rio de Janeiro" - 5 p. Rio de Janeiro. 1884. Tip. Nacional.
Araújo, Antônio Bahia da Silva - Meios de desenvolver a instrução primária nos municípios rurais. In "Atas e Pareceres do Congresso de Instrução do Rio de Janeiro " - 4 p. Rio de Janeiro. 1884. Tip. Nacional.

Azevedo, Manuel Antônio Duarte Moreira de - $\mathrm{O}$ regime de Internato nos estabelecimentos de instrução secundária $\mathrm{e}$ nas escolas normais. In "Atas e Pareceres do Congresso de Instrução do Rio de Janeiro " -3 p. Rio de Janeiro. 1884. Tip. Nacional.

Barcelos, Licinio Chaves - Sistema disciplinar e meios e emulação para os alunos dos estabelecimentos de ensino superior. In "Atas e Pareceres do Congresso do Rio de Janeiro " - 3 p. Rio de Janeiro. 1884. Tip. Nacional.

Barreto, Rosendo Monis (Dr.) - Sistema disciplinar e meios de emulação nas escolas de diferentes graus. In "Atas e Parecercs do Congresso de Instrução do Rio de Janeiro " - 13 p. Rio de Janeiro. 1884. Tip. Nacional.

Bittencourt, Feliciano Pinheiro de Educação dos cegos - In "Atas e Pareceres do Congresso de Instrução Rio de Janeiro" - 2 p. - Rio de Janeiro. 1884. Tip. Nacional.

Brandão, Alberto - Sistema disciplinar e meios de emulação nas escolas de diferentes graus. In "Atas e Parecercs do Congresso de Instrução do Rio de Janeiro " -2 p. - Rio de Janeiro. 1884. Tip. Nacional.

Caminhoá. Joaquim Monteiro (Cons.) Sistema disciplinar e meios de emulação para os alunos dos estabelecimentos de ensino superior. In "Atas c Pareceres do Congresso de Instrução do Rio de Janeiro" - 10 p. Rio de Janeiro. 1884. Tip. Nacional. Carreira, José Aldrete de Mendonça Rangel de Queiroz - Mesa de exa- 
mes gerais de preparatórios na corte. Concessão aos estabelecimentos de instrução secundária de prerrogativas para validade de exames. In "Atas e Pareceres do Congresso de Instrução do Rio de Janeiro " - 5 p. Rio de Janeiro. 1884. Tip. Nacional.

Castro, A. V. de Borja - Estado do ensino superior. Vícios e lacunas. In "Atas e Pareceres do Congresso de Instrução do Rio de Janeiro " - 11 p. Rio de Janeiro. 1884. Tip. Nacional.

Cavalcanti, Amaro - Meios de desenvolver a instrução primária nos municípios rurais. In "Atas e Pareceres do Congresso de Instrução do Rio de Janeiro "- 15 p. Rio de Janeiro. 1884. Tip. Nacional.

Cavalcanti, João Barbalho Uchoa (Dr.) Coeducação dos sexos nas escolas primárias, normais e secundárias. In "Atas e Pareceres do Congresso de Instrução do Rio de Janeiro - 12 p. Rio de Janeiro. 1884. Tip. Nacional.

Cavalcanti, João Barbalho Uchoa - Meios de desenvolver a instrução primária nos municípios rurais. In "Atas e Pareceres do Congresso de Instrução do Rio de Janeiro " - 10 p. Rio de Janeiro. 1884. Tip. Nacional.

Cony, Auguslo Cândido Xavier - Sistema disciplinar e meios de emulação nas escolas de diversos graus. In "Atas e Pareceres do Congresso de Instrução do Rio de Janeiro " - 17 p. Rio de Janeiro. 1884. Tip. Nacional.

Cordeiro, Lopo Dinis (Dr.) — Ensino de Moral e Religião nas Escolas Primárias, nos Estabelecimentos de instrução secundária e nas Escolas Normais. In "Atas e Pareceres do Congresso de Instrução do Rio de Janeiro " - 5 p. Rio de Janeiro. 1884. Tip. Nacional.
Costa e Cunha, Antônio Estêvão da - A Educação Física nos Jardins da Infância, Escolas Primárias e Colé gios. In "Atas e Pareceres do Con gresso de Instrução do Rio de Ja neiro " - 20 p. Rio de Janeiro. 1884. Tip. Nacional.

Costa, Manuel Olímpio Rodrigues do - Classificação das Escolas Primá rias. Disciplinas que devem ser ensi nadas. Material escolar. In "Atas $e$ Pareceres do Congresso de Instrução do Rio de Janeiro " - 12 p. Rio de Ja neiro. 1884. Tip. Nacional.

Costa, Manuel Olímpio Rodrigues da

- Métodos e Programas de Ensino nas Escolas Primárias. Adoção de compêndios. In "Atas e Pareceres do Congresso de Instrução do Rio de Janeiro" — 5 p. Rio de Janeiro. 1884. Tip. Nacional.

Dantas. Rodolfo Epifânio de Sousa (Cons.) - Ensino de Moral e Religião nas Escolas Primárias, Secundárias e Normais. In "Atas e Pareceres do Congresso de Instrução do Rio de Janeiro " - 12 p. Rio de Janeiro. 1884. Tip. Nacional.

Esberard, João (Monsenhor) --- Criação de uma faculdade de ciências religiosas. Sua organização. In "Atas e Pareceres do Congresso de Instrução do Rio de Janeiro" - 14 p. Rio de Janeiro. 1884. Tip. Nacional.

Figueiredo, Afonso Celso de Assis (Cons.) - Competência dos poderes gerais para criar estabelecimentos de

ensino primário e secundário nas províncias. In "Atas c Pareceres do Congresso de Instrução do Rio de Janeiro" - 8 p. Rio de Janeiro. 1884. Tip. Nacional.

Figueiredo Júnior, Afonso Celso de Assis Liberdade do ensino superior. In "Atas $e$ Pareceres do Congresso de 
REVISTA BRASILEIRA DE ESTUDOS PEDAGÓGICOS

Instrução do Rio de Janeiro " - 8 p. Rio de Janeiro. 1884. Tip. Nacional.

Figueiredo, José Bento da Cunha, Medidas conducentes a tornar efetiva a inspeção do ensino. In "Atas e Pa-receres do Congresso de Instrução do Rio de Janeiro" - 3 p. Rio de Janeiro. 1884. Tip. Nacional.

Figueiredo, José Bento da Cunha Concessão aos estabelecimentos de instrução secundária de prerrogativas para validade de exames, mediante certas garantias. In "Atas e Pareceres do Congresso de Instrução do Rio de Janeiro" - 3 p. Rio de Janeiro. 1884. Tip. Nacional.

Figueiredo, José Bento da Cunha Concessão, mediante certas garantias. da prerrogativa de que goza o Imperial Colégio de Pedro II aos estabelecimentos de instrução secundária. In " Atas e Pareceres do Congresso de Instrução do Rio de Janeiro ". Rio de Janeiro. 1884. Tip. Nacional.

Figueiredo, José Bento da Cunha - Mesas de exames gerais da Corte: processo dos exames. Exames nas províncias. In "Atas e Pareceres do Congresso de Instrução do Rio de Janeiro" - 3 p. Rio de Janeiro. 1884. Tip. Nacional.

Filgueiras Júnior, Araújo - Parecer (sobre mesas de exames gerais de preparativos). Rio de Janeiro. J884. (S/Edit.). 36 p.

França. Ernesto Ferreira - Competência dos poderes gerais para criar estabelecimentos de ensino primário e secundário nas províncias. In "Atas $e$ Pareceres do Congresso de Instrução do Rio de Janeiro" — 34 p. Rio de Janeiro. 1884. Tip. Nacional.

Frazão, Manuel José Pereira - Organização das bibliotecas e museus escolares e pedagógicos. Caixas econômi- cas escolares. In "Atas e Pareceres do Congresso de Instrução do Ria de Janeiro. 1884. Tip. Nacional.

Frasão, Rosalina - Classificação das Escolas Primárias. Disciplinas aue devem ser ensinadas. Material escolar. In " Atas $e$ Pareceres do Congresso de Instrução do Rio de Janeiro" - 10 p. Rio de Janeiro. 1884. Tip. Nacional.

Freitas, Antônio de Paula - Escola Politécnica. Cursos especiais. In "Atas e Pareceres do Congresso de Instrução do Rio de Janeiro" - 11 p. Rio de Janeiro. 1884. Tip. Nacional.

Frontin, André Gustavo Paulo de Organização e atribuições do conselho diretor da instrução primária e secundária do município da corte. In "Atas e Pareceres do Congresso da Instrução do Rio de Janeiro" - 5 p. Rio de Janeiro. 1884. Tip. Nacional.

Cama, José de Saldanha da - Escola Politécnica. Cursos especiais. In " Atas e Pareceres do Congresso de Instrução do Rio de Janeiro" - 10 p. Rio de Janeiro. 1884. Tip. Nacional.

Garcia, José Manuel - Classificação das Escolas Primárias. Disciplinas que devem ser ensinadas. In "Atas e Pareceres do Congresso de Instrução do Rio de Janeiro " - 11 p. Rio de Janeiro. 1884. Tip. Nacional.

Garcia, José Manuel - Educação dos adultos e das adultas. In "Atas e Pareceres do Congresso de Instrução do Rio de Janeiro "'- 9 p. Rio de Janeiro. 1884. Tip. Nacional.

Garcia. José Manuel (Dr.) - Métodos e Programas do Ensino na Escola Primária. Sua reforma. Adoção de livros. In "Atas e Pareceres do Congresso de Instrução do Rio de Janeiro" - 10 p. Rio de Janeiro. 1884. Tif. Nacional. 
Guimarães, Jose Pereira — Faculdade de Medicina. Cursos especiais. In "Atas e Pareceres do Congresso de Instrução do Rio de Janeiro" - 6 p. Rio de Janeiro. 1884. Tip. Nacional.

Itapoan, Barão de - Faculdade de Medicina. Cursos especiais. In "Atas $e$ Pareceres do Congresso de Instrução do Rio de Janeiro " - 35 p. Rio de Janeiro. 1884. Tip. Nacional.

Jaguari, Visconde de - Competência dos poderes provinciais para criar estabelecimentos de ensino superior. In "Atas e Pareceres do Congresso de Instrução do Rio de Janeiro " - 2 p. Rio de Janeiro. 1884. Tip. Nacional.

Laet, Carlos Maximiano Pimenta de Criação de uma faculdade de letras. Sua organização. In "Atas e Pareceres do Congresso de Instrução do Rio de Janeiro" — 19 p. Rio de Janeiro. 1884. Tip. Nacional.

Laet. Carlos Maximiano Pimenta - -Escolas Normais. Sua organização, plano de estudo, métodos e programas de ensino. In " Atas e Pareceres do Congresso de Instrução do Rio de Janeiro " - 15 p. Rio de Janeiro. 1884. Tip. Nacional.

Leal, Antônio Henrique (Dr.) - Higiene Escolar. In "Atas e Pareceres do Congresso de Instrução do Rio de Janeiro" - 6 p. Rio de Janeiro" - 6 p. Rio de Janeiro. 1884. Tip. Nacional.

Leão, Teófilo das Neves - Coeduca-ção dos sexos nas escolas primárias, nos estabelecimentos de instrução secundária e nas escolas normais. In "Atas e Pareceres do Congresso de Instrução do Rio de Janeiro " -2 p. Rio de Janeiro. 1884. Tip. Nacional.

Leitão, Antônio Cândido da Cunha (Dr.) Ensino Primário Obrigató- ria. Meios de torná-lo efetivo. In "Atas c Pareceres do Congresso de Instrução do Rio de Janeiro." - 21 p. Rio de Janeiro. 1884. Tip. Nacional.

Leitão, Antônio Cândido da Cunha -Liberdade do ensino primário e secundário. In "Atas e Pareceres do Congresso de Instrução do Rio de Janeiro " - 17 p. Rio de Janeiro. 1884. Tip. Nacional.

Leme, Pedro Dias Gordilho Pais Educação dos adultos e das adultas. In "Atas e Pareceres do Congresso de Instrução do Rio de Janeiro " - 2 p. Rio de Janeiro. 1884. Tip. Nacional.

Leme, Pedro Dias Gordilho Pais - Escolas profissionais e de aprendizado. Sua organização. In "Atas e Pareceres do Congresso de Instrução do Rio de Janeiro " - 5 p. Rio de Janeiro. 1884. Tip. Nacional.

Lima. Agostinho Jose de Sousa Faculdades livres, suas prerrogativas. In "Atas e Pareceres do Congresso de Instrução do Rio de Janeiro " - 10 p. Rio de Janeiro. 1884. Tip. Nacional

Macedo, José Teixeira de (Dr.) Organização dos Jardins de Infância. In "Atas e Pareceres do Congresso de Instrução do Rio de Janeiro " - 16 p. Rio de Janeiro. 1884. Tip. Nacional.

Magalhães, Benjamim Constant Botelho de - Escolas Normais. Sua organização, plano de estudo, métodos e programas de ensino. In "Atas e Pareceres do Congresso de Instrução do Rio de Janeiro" - 36 p. Rio de Janeiro. 1884. Tip. Nacional,

Maia, João Carlos de Oliva - O regime de Internato nos estabelecimentos de instrução secundária e nas escolas normais. In "Atas e Pareceres do Congresso de Instrução do Rio de Janeiro" - 13 p. Rio de Janeiro. 1884. Tip. Nacional. 
Melo, Homem de (Barão) - $\mathrm{O}$ regime de internato nos estabelecimentos de instrução secundária e nas escolas normais. In "Atas e Pareceres do Congresso de Instrução do Rio de Janeiro " - 3 p. Rio de Janeiro. 1884. Tip. Nacional.

Monteiro. João Pereira - Processo e julgamento dos exames nos estabelecimentos de ensino superior. In "Atas e Pareceres do Congresso de Instrução do Rio de Janeiro" - 4 p. Rio de Janeiro. 1884. Tip. Nacional.

Melo, João Capistrano Bandeira de Criação de uma faculdade de ciências religiosas. Sua organização. In "Atas $e$ Pareceres do Congresso de Instrução do Rio de Janeiro" — 9 p. Rio de Janeiro. 1884. Tip. Nacional.

Morais, J. de A. Leite - Sistema disciplinar e meios de emulação para os alunos dos estabelecimentos de ensino superior. In "Atas e Pareceres do Congresso de Instrução do Rio de Janeira " - 7 p. Rio de Janeiro. 1884. Tip. Nacional.

Nogueira. Manuel Tomas Alves - Imperial Colégio de Pedro II. Sua organização. In "Atas e Pareceres do Congresso de Instrução do Rio de Janeiro " - 6 p. Rio de Janeiro. 1884. Tip. Nacional.

Nogueira, M. T. Alves - Organização do ensino secundário para o sexo feminino. In "Atas e Pareceres do Congresso de Instrução do Rio de Janeiro " - 8 p. Rio de Janeiro. 1884. Trip. Nacional.

Oliveira. A. de Almeida - Criação de uma universidade. Sua organização. Organização do conselho universitário. In "Atas e Pareceres do Conselho de Instrução do Rio de Janeiro" - 20 p. Rio de Janeiro. 1884. Tip. Nacional. Oliveira, Álvaro Joaquim (Dr.) -
Processo e julgamento dos exames nos estabelecimentos de ensino superior. In " $A$ tas e Pareceres do Congresso de Instrução do Rio de Janeiro" - 7 p. Rio de Janeiro. 1884. Tip. Nacional.

Otôni, Cristiano Benedito (Cons.) - Estado do ensino superior. Vícios e lacunas. In "Atas e Pareceres do Congresso de Instrução do Rio de Janeiro" — 7 p. Rio de Janeiro. 1884. Tip. Nacional.

Passos. Francisco Pereira - Cursos livres nos estabelecimentos de ensino superior. In "Atas e Parecer es do Congresso de Instrução do Rio de Janeiro" - 6 p. Rio de Janeiro. 1884. Tip. Nacional.

Pereira, Antônio Pacífico - Faculbelecimentos de ensino superior. In "Alas e Pareceres do Congresso de Instrução do Rio de Janeiro" - 25 p. Rio de Janeiro. 1884. Tip. Nacional.

Pereira, João Batista - Cursos livres nos estabelecimentos de ensino superior. In "Alas e Pareceres do Congresso de Instrução do Rio de Janeiro " - 8 p. Rio de Janeiro. 1884. Tip. Nacional.

Pitanga. Epifànio Cândido de Sousa Organização do professorado dos estabelecimentos de ensino superior. In "Atas $e$ Pareceres do Congresso de Rio de Janeiro ". 1884. Tip. Nacional.

$11 p$

Pisam Pilha, Teresa - Organização do ensino secundário para o sexo feminino. In "Atas e Pareceres do Congresso de Instrução do Rio de Ja-neiro " - 11 p. Rio de Janeiro. 1884. 'Tip. Nacional.

Prado, Manuel José de Meneses - Medidas conducentes a tornar efetiva a inspeção do ensino primário e secundário. In "Alas $e$ Pareceres do Con- 
gresso de Instrução do Rio de Janeiro" — 7 p. Rio de Janeiro. 1884. Tip. Nacional.

Rebouças, André - Escolas profissionais e de aprendizado. Sua organização. In "Atas e Pareceres do Congresso de Instrução do Rio de Janeiro " - 4 p. Rio de Janeiro. 1884. Tip. Nacional.

Reis, Manuel Pereira (Dr.) - Escola Politécnica. Cursos especiais. In "Atas $e$ Pareceres do Congresso do Rio de Janeiro " -- 4 p. Rio de Janeiro. 1884. Tip. Nacional.

Ribas, Antônio Joaquim (Cons.) - Criação de uma universidade. Sua organização. Organização do conselho universitário. In "Atas e Pareceres do Congresso de Instrução do Rio de Janeiro " - 2 p. Rio de Janeiro. 1884. Tip. Nacional.

Rodrigues. A. Coelho (Dr.) - Cursos livres nos estabelecimentos de ensino superior. In "Atas e Pareceres do Congresso de instrução do Rio de Janeiro" - 5 p. Rio de Janeiro. 1884. Tip. Nacional.

Romero, Silvio (Dr.) - Ensino Primário Obrigatório - Meios de toma-, Io efetivo. In "Atas e Pareceres do Congresso de Instrução do Rio de Janeiro " - 2 p. Rio de Janeiro. 1884. Tip. Nacional.

Romero, Sílvio (Dr.) — Liberdade de Ensino Primário e Secundário. In "Atas e Pareceres do Congresso de Instrução do Rio de Janeiro" 3 p. Rio de Janeiro. 1884. Tip. Nacional.

Sabino, Januário dos Santos - Classificação das Escolas Primárias. Disciplinas que devem ser ensinadas. In " Atas e Pareceres do Congresso de Instrução do Rio de Janeiro" 7 p. Rio de Janeiro. 1884. Tip. Nacional.
Sabino. Januário dos Santos - Métodos e Programas do Ensino nas Escolas Primárias e nos estabelecimentos de instrução Secundária. In "Atas c Pareceres do Congresso de Instrução do Rio de Janeiro" - 10 p. Rio de Janeiro. 1884. Tip. Nacional.

Sabóia, Vicente (Dr.) - Faculdade de Medicina. Cursos especiais. In "Atas e Pareceres do Congresso de Instrução do Rio de Janeiro" — 9 p. Rio de Janeiro. 1884. Tip. Nacional.

Silva, Francisco Rodrigues da (Cons.) - Coeducação dos sexos nos estabelecimentos de ensino superior. In "Atas e Pareceres do Congresso de Instrução do Rio de Janeiro" - 6 p. Rio de Janeiro. 1884. Tip. Nacional. Silva, Francisco Rodrigues (Cons.)

- Estado do ensino superior. Vícios e lacunas. In "Atas e Pareceres do Congresso de Instrução do Rio de Janeiro" - 16 p. Rio de Janeiro. 1884. Tip. Nacional.

Silva, Josino do Nascimento - Medidas conducentes a tornar efetiva a inspeção do ensino. In "Atas e Pareceres do Congresso de Instrução do Rio de Janeiro" - 3 p. Rio de Janeiro. 1884. Tip. Nacional.

Souto, L. R. Vieira - Caixas econômicas escolares. In "Atas e Pareceres do Congresso de Instrução do Rio de Janeiro" - 46 p. Rio de Janeiro. 1884. Tip. Nacional.

Sousa, Enes de - Escola de Minas de Ouro Preto. Sua organização e ensino. In "Atas e Pareceres do Congresso de Instrução do Rio de Janeiro" - 7 p. Rio de Janeiro. Tip. Nacional.

Vieira, Menezes (Dr.) - Organização dos Jardins de Infância. In "Atas e Pareceres do Congresso de Instrução do Rio de Janeiro" - 10 p. Rio de Janeiro. 1884. Tip. Nacional. 
REVISTA BRASILEIRA DE ESTUDOS PEDAGÓGICOS

Vieira. Menezes - Organização do professorado para os jardins da infância e para o ensino primário e secundário. In "Alas e Pareceres do Congresso de Instrução do Rio de Janeiro " - 7 p. Rio de Janeiro. 1884. Tip. Nacional.

AzAmbuja, Conselheiro de - Doutrinas Pedagógicas e Elementos de Instrução Pública. Pará. 1884. João Cardoso \& Cia. 126 p.

Relatório acompanhado de considerações gerais sobre o ensino na Província do Amazonas.

CAVAlCANTI. João Barbalho Uchoa Instrução Pública. Pernambuco. 1884. Manuel Figueiroa de Faria \& Cia. 164 p.

Relatório sobre o ensino primário da província.

Conferências efetuadas na Exposição Pedagógica - Por Carlos Leôncio de Carvalho, Antônio de Paula Freitas, Joaquim .Abilio Borges, Amaro Cavalcanti e Abílio César Borges. Rio de Janeiro. 1884. Tip. Nacional. 210 p.

Borges, Abílio César - Sobre o Ensino no Colégio Abílio. Pg. 183 a 203 de: Conferências efetuadas na Exposição Pedagógica. Rio de Janeiro. 1884. Tip. Nacional. $210 \mathrm{p}$.

Borges, Joaquim Abílio - Exposição do Colégio Abílio. Pg, 45 a 110 de: Conferências efetuadas na Exposição Pedagógica. Rio de Janeiro. 1884. Tip. Nacional. 210 p.

Carvalho, Carlos Leôncio de (Cons.) Educação da Infância Desamparada. Pg. 6 a 19 de: Conferências efetuadas na Exposição Pedagógica. Rio de Janeiro. 1884, Tip. Nacional. 210 p.
Cavalcanti. Amaro - O ensino moral e religioso nas' Escolas Públicas. Pg. 113 a 137 de: Conferências efetuadas na Exposição Pedagógica. Rio de Janeiro. 1884. Tip. Nacional. $210 \mathrm{p}$.

Freitas, Antônio de Paula (Dr.) - Higiene Escolar. Pg. 27 a 42 de: Conferências efetuadas na Exposição Pedagógica. Rio de Janeiro. 1884. Tip. Nacional. 210 p.

Macaúbas (Barão de) - Aparelho Escolar Múltiplo, Pg. 14,1 a 181 de: Conferências efetuadas na Exposição Pedagógica. Rio de Janeiro. 1884. Tip. Nacional. 210 p.

DÓRIA, Franklin - Museu Escolar Nacional. Rio de Janeiro. 1884. Tip. Nacional. 8 p.

Discurso com que o Cons. Franklin Dória fundamentou na Câmara dos Deputados um projeto de sua autoria sobre a criação de um Museu Escolar Nacional.

Exposição (Primeira) Pedagógica do Rio de Janeiro — Rio de Janeiro. 1884. Tip. Nacional. Seção Histórica e Seção Filosófica - 259 p. Comissão Diretora, da Exposição Pedagógica. Atas - 88 p. Júri da Exposição Pedagógica - 104 p. Pare-ceres - $240 p$.

Alas. Pareceres e Discursos. Discriminam-se a seguir os dois trabalhos aqui reunidos.

Carvalho. Leôncio - Seção Histórica e Seção Filosófica. 259 p. de: Exposição Pedagógica (Primeira) do Rio de Janeiro. Rio de Janeiro. 1884. Tip. Nacional.

Relatório do Secretário da Comissão organizadora da Exposição Pedagógica. 
Comissão Diretora da Exposição Pedagógica (1. $\left.{ }^{\circ}\right) .88$ p. de: Exposição Pedagógica (Primeira) do Rio de Janeiro. Rio de Janeiro. 1884. Tip. Nacional.

Transcrição das atas e trabalhos da Comissão referida, que foi presidida pelo Conde d'Eu e tinha como membros o Visconde de Bom Retiro, e os Conselheiros Manuel Francisco Correia. Carlos Leôncio de Carvalho e Franklin Américo de Menezes Dória.

MElo, Alfredo Pinto Vieira de - Educação Científica. Recife. 1884. Tip. R. de Flores. $24 p$.

Vulgarização de princípios filosóficos. científicos e pedagógicos.

Pedagogia - Rio de Janeiro. 1884. Tip. Nacional. $84 \mathrm{p}$

Trabalho da $8^{a}$ Conferência Pedagógica dos Professores Públicos Primários dos Municípios da Corte.

LiMA, César Augusto Viana (Dr.) - O Ensino Primário no Reino Unido da Grã-Bretanha e Irlanda. Rio de Janeiro. 1885. Imprensa Nacional. $98 \mathrm{p}$

Estudo sobre o ensino primário no Reino Unido da Grã-Bretanha $e$ Irlanda.

AlmeIDA, Pires (Dr.) - Oficina na Escola. Rio de Janeiro. 1886. Lam-baerts \& Cia. $24 \mathrm{p}$.

Notícia histórica do ensino profissional pôsto em prática em escolas elementares do Brasil, pelo Imperador Pedro II.

CAVAlCANTI, João Barbalho Uchoa Instrução Pública. Pernambuco.
1886. Manuel Figueiroa de Faria \& Filhos. 166 p

Relatório sobre a situação do ensino primário, normal e secundário nessa Província.

Borges, Abílio César - Educação c Ensino. Paris. 1886. Livr. da "Vve. J. P. Aillaud Guillard \& Cia". (S/N. ${ }^{\circ}$ p.).

Discursos.

CAlkins, $N$. S. - Primeiras Lições de Coisas. Trad. da $40^{\circ}$ Ed. amer. por Rui Barbosa. Rio de Janeiro. 1886. Impr. Nacional. 613

Manual de ensino elementar para uso dos pais e professores. Uso de processos intuitivos. Metodologia.

LEITÃo, Antônio Cândido da Cunha (Dr.) - O Ensino Primário e Secundário em um " Plano Nacional de Ensino". Rio de Janeiro. 1886. Impr. Nacional. (S/n. ${ }^{\circ}$ p.)

Bases para a reorganização do Ensino Primário e Secundário no Município Neutro, desenvolvimento da Instrução Pública nas Províncias c elevação do ensino secundário em todo o Império.

Luz, J. C. D'Alambari - Trabalho da 9. Conferência Pedagógica dos Professores Públicos Primários da Corte. Rio de Janeiro. 1886. Impr. Nacional. 217 p.

Brazilicus - Guia Pedagógica de Cálculo Mental e Uso do Contador Mecânico ou Aritmômetro no ensino elementar da Aritmética. Rio de Janeiro. 1887. Livr. Clássico de Alves \& Cia. 198 p.

Metodologia do ensino da aritmética. 
Jaguaribe Filho, Domingos José Nogueira (Dr.) FERrão. Maria C. Gomes — Educação Física. - Inteligência e Moral do Homem. São Paulo. 1887. Tip. King. 170 p. (Da Ginástica). Bahia. 1890. Impr. Popular. $112 p$

Principios da educação intelectual $c$ moral.

Hacilman, $W . W$. - Jardim das Crianças. Trad. de G. L. A. Rio de Janeiro. 1887. S/ Edit. LEMos, Miguel — A Obrigatoriedade do $\left(S / n .^{o} p\right.$.).

Exposição do sistema de Froebel.

PASSAláQUA, Camilo - Pedagogia e Metodologia (Teoria e Prática). São Paulo. 1887. Tip. de Jorge Se-ckler \& Cia. 179 p.

Compendio para as escolas normais.

SousA Filho, Tarquinio de - O Ensino técnico Janeiro. 1890. Tip. Montenegro. (S/n. $\left.{ }^{o} p.\right)$. no Brasil. Rio de Janeiro. 1887. Impr. Nacional. $243 p$.

$$
\text { A situação do ensino no Brasil e }
$$
especialmente a do ensino técnico.

CARvalho, Felisberto Rodrigues Pereira de Tratado de Metodologia Coordenada. Rio de Janeiro. 1888. Alves \& Cia. 218 p.

$O$ A., que foi um dos renovadores do ensino primário no fim do século passado, expõe suas idéias sobre a orientação a ser dada à instrução elementar. ensino. Rio de Janeiro. 1890. Ev. da Costa. $2 p$.

Exposição da doutrina positivista em relação à obrigatoriedade do ensino.

RIBEIRO, João - A Instrução Pública

Memória escrita para o concurso ao lugar de Diretor da Instrução Pública do Estado de Minas Gerais.

Veríssimo, José - A Educação Nacional. Pará. 1890. Livr. Universal. 182 p.

Coletânea de estudos vários, versando o tema central sobre a necessidade de organizar-se a educação do pais com sentido nacional.

\section{A 1900}

CAVALCANTI, João Barbalho Uchoa Instrução Pública. Rio de Janeiro. 1891. Impr. Nacional. 136 p. c/anexos.

\section{Relatório.}

\section{Conferência.}

Romero, Silvio - A Filosofia e o Ensino CoElHo, J. Augusto - Princípios de Secundário. Rio de Janeiro. 1889. Centro Bibliográfico. $26 \mathrm{p}$.

Critica ao ensino da cadeira de filosofia do Imperial Colégio Pedro II.

Pedagogia. São Paulo. 1891/1893.

Teixeira \& Irmãos. 4 tomos em 2 rols. cl 445 e $601 f$

Tratado de pedagogia geral, calcado sobre o livro de Spencer " A Educação Física, Intelectual e Moral". 
VERÍSSIMO, José - A Instrução Pública no Estado do Pará em 1890. Pará.

1891. S/ Edit. 205 p.

Relatório apresentado ao Governador do Pará pelo Diretor Geral de Instrução Pública do Estado.

PREstes, Gabriel - A Reforma do Ensino Público. São Paulo. 1892. Le-roy King. $102 \mathrm{p}$.

Coletânea de artigos publicados na imprensa sobre reformas do ensino primário.

ReIS, Luís Augusto - O Ensino Público Primário em Portugal, Espanha, França e Bélgica. Rio de Janeiro. 1892. Impr. Nacional. $650 \mathrm{p}$.

Relatório apresentado à Inspetoria Geral da Instrução Pública da Capital federal.

VIEIRA, Menezes - O Pedagogium Brasileiro. Rio de Janeiro. 1892. Tip. Camões. $\left(S / n .^{\circ} p\right.$.).

Notícia para a Exposição Universal Colombiana de Chicago.

Costa, Amélia Fernandes da - O Ensino Público Primário na Itália, França e Bélgica. Rio de Janeiro.

1893. Impr. Nacional. $148 p$.

Relatório apresentado à Inspetoria Geral de Instrução Primária e Secundária da Capital Federal.

Frazão, Manuel José Pereira - O Ensino Público Primário. Rio de Janeiro. 1893. Tip. da "Gazeta de Noticias". 516 p. c/gravuras.

Relatório apresentado à Inspetoria Geral de Instrução Primária da Capital Federal.
CAldas, A. e Carvalho, E. - Manual de Ginástica Escolar. Rio de Janeiro. 1896. Livr. Francisco Alves. 230 p.

Guia para uso dos professores.

EstradA, L. Duque e FrEIRE, Laudelino In " Revista do Instituto Didático". Vol. I 1895. Rio de Janeiro.

Noticia sobre a evolução do ensino no Brasil.

Higgins, A. - Compêndio de Ginástica e Jogos Escolares. Rio de Janeiro. 1896. Tip. do Jornal do Comércio. 137 p.

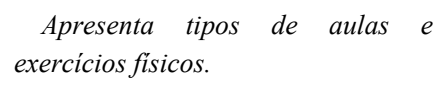

Pimentel, T. Mendes - Organização do Ensino Profissional Primário no Estado de Minas Gerais. Ouro Preto. 1896. Impr. Oficial de Minas Gerais. 26 p.

Justificação de um projeto de lei sobre o ensino profissional em grau elementar.

PORTOCARRERO, Leopoldina Tavares - O ensino público primário em França, Espanha e Portugal. Rio de Janeiro. 1896. Ofs. do Instituto Profissional. ( $\mathrm{S} / \mathrm{n} .^{\circ} \mathrm{p}$.).

Relatório de uma viagem de estudos.

RENOTE, Marie - Influência da educação da mulher sobre a medicina social. Rio de Janeiro. 1896. Tip. " Aldina". 29 p.

Tese apresentada à Faculdade de Medicina e Farmácia do Rio de Janeiro.

WERnECK, Américo — Arte de Educar os Filhos. Rio de Janeiro. 1896. Empr. Graf. Edit. Paulo Pongetti \& Cia. 242 p. 
Conselhos sobre puericultura. $\quad$ Silvado, Brasil - Educação dos Cegos. Rio

apresentado sob a forma de cartas.

Gomes, Alfredo - Ensino Municipal. Rio de Janeiro, 1897. Rodrigues \& Cia. 82 p.

Crítica e comentário ao decreto de 9 de abril de 1897, que reformou o ensino municipal do Distrito Federal. de Janeiro. 1897. Impr. Nacional. 48 p.

Guia prático para o ensino da leitura aos cegos.

Pitombo, A. C. - Apreciação acerca dos exercícios físicos nos internatos e sua importância profilática. S/Ed.. Bahia, 1900. $\left(S / n .^{\circ}\right.$ p.). 